\title{
Evaluation of the Prognostic Value of Chest Computed Tomography Scan in COVID-19 Patients
}

\author{
Behzad Aminzadeh (iD ${ }^{1}$, Parvaneh Layegh ${ }^{1}$, Mahdi Foroughian (iD $^{2}$, Ahmadreza Tavassoli (iD) ${ }^{1}$, Maryam

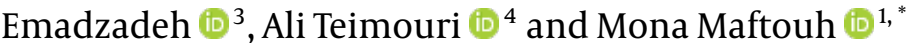 \\ ${ }^{1}$ Department of Radiology, Faculty of Medicine, Mashhad University of Medical Sciences, Mashhad, Iran \\ ${ }^{2}$ Department of Emergency Medicine, Faculty of Medicine, Mashhad University of Medical Sciences, Mashhad, Iran \\ ${ }^{3}$ Department of Community Medicine, Faculty of Medicine, Mashhad University of Medical Sciences, Mashhad, Iran \\ ${ }^{4}$ Student Research Committee, Mashhad University of Medical Sciences, Mashhad, Iran \\ "Corresponding author: Department of Radiology, Faculty of Medicine, Mashhad University of Medical Sciences, Mashhad, Iran. Email: monarch.1171@gmail.com
}

Received 2020 October 18; Revised 2021 June 09; Accepted 2021 June 12.

\begin{abstract}
Background: The world is facing the coronavirus 2 pandemic since 2019 (COVID-19 infection) and all countries have challenges in management of patients based on their facilities. Chest computed tomography (CT) scan can be valuable in early detection and estimation of the pulmonary involvement in these patients.

Objectives: To evaluate the prognostic value of chest CT imaging features in patients with coronavirus disease 2019(COVID-19) pneumonia.

Patients and Methods: In this cross-sectional study, 201 patients with COVID-19 were enrolled consecutively. The patients' chest CT scans were analyzed, and the disease severity was rated using two methods: (1) total lung involvement (TLI) in which each lobe is scored from 0 to 4 based on the percentage of involvement; and (2) modified TLI in which each lobe involvement score is multiplied by the number of its segments, and the sum is recorded as the modified TLI. The patients were categorized into four groups depending on their prognosis (patients admitted to hospital wards, patients admitted to intensive care units [ICUs], patients with intubation during hospitalization, and expired patients). The relationship between both scoring methods and the clinical outcomes of patients was examined in the four groups.

Results: The receiver operating characteristic (ROC) curve analysis showed no significant difference between the two scoring methods (TLI and modified TLI) in predicting the patients' prognosis. The average disease severity based on the two scoring methods was significantly different between the four groups. Patients who were intubated during hospitalization and patients who expired had significantly higher scores than patients admitted to the ICUs and hospital wards $(\mathrm{P}=0.001)$. The area under the ROC curve for the prediction of mortality was 0.81 (95\% CI: $0.72-0.90 ; \mathrm{P}<0.001$ ). The TLI score of 18.5 could predict mortality with specificity of $>95 \%$. Conclusion: The TLI scoring system can be used for predicting in-hospital mortality and ICU admission in COVID-19 patients. This scoring method can help us devise a better strategic healthcare plan during the COVID-19 pandemic.
\end{abstract}

Keywords: COVID-19, Spiral Computed Tomography, Prognosis

\section{Background}

A new member of coronavirus family, called severe acute respiratory syndrome coronavirus 2 (SARS-CoV-2), is responsible for the new emerging viral pneumonia, known as coronavirus disease 2019 (COVID-19) (1-3). Today, real-time reverse transcription-polymerase chain reaction (RT-PCR) of respiratory tract specimens is known as the reference standard for diagnosis of SARS-CoV-2 infection (4). Due to different intrinsic limitations, such as sampling, transportation, and diagnostic kit performance, the sensitivity of RT-PCR for detecting COVID-19 ranges from 60 to $71 \%$ in the initial presentation (5-8).
Old age and comorbidities, such as cardiovascular and pulmonary diseases and diabetes, have been recently proposed as prognostic factors for COVID-19, causing more complications and exacerbating the disease (9-11). According to previous studies, laboratory findings and biomarkers, such as C-reactive protein (CRP), D-dimer, CD8 T lymphocyte count, N-terminal prohormone of brain natriuretic peptide (NT-proBNP), interleukin-6 (IL-6), and procalcitonin may play a prognostic value in COVID-19 $(9,10$, $12,13)$.

Chest computed tomography (CT) scan plays an increasingly important role in the early detection of COVID- 
19. It is used to estimate the clinical course and outcomes of COVID-19 in patients (14). A recent study showed that the well-aerated lung (WAL) percentage, assessed by chest CT scan upon admission, is correlated with improved clinical outcomes in COVID-19 patients. To give an example, patients with WAL $<73 \%$ were 5.4 times more likely to be admitted to the intensive care unit (ICU) or expire (15).

\section{Objectives}

This study aimed to evaluate the prognostic significance of quantitative chest CT scan in COVID-19 patients. We analyzed the patients' initial chest CT scans and scored the lung involvement using two methods:

- Total lung involvement score (TLI): Each lobe was scored from 0 to 4 based on the percentage of involvement, according to a study by Hani et al. (16).

- Modified TLI: Each lobe involvement was first multiplied by the number of lobe segments, and the sum was suggested as the modified TLI. The correlation between the recorded scores and outcomes of COVID-19 was evaluated in four groups: patients admitted to hospital wards, patients admitted to the ICU, patients intubated during hospitalization, and expired patients.

\section{Patients and Methods}

\subsection{Study Participants}

This cross-sectional study was conducted according to the principles of the declaration of Helsinki and our institutional review board (IR.MUMS.REC.1399.029). No informed consent form was obtained from the participants because of the retrospective design of this study. Patients with laboratory-identified COVID-19 based on real-time RTPCR were included in this study between May 2020 and June 2020. The clinical data included age, sex, and admission ward. Patients with pathological findings other than those related to COVID-19 in chest CT scans (e.g., pattern of lung involvement compatible with tuberculosis and pulmonary fibrosis) were excluded from the study; also, patients with missing data were eliminated.

\subsection{Chest CT Scan}

We used the available chest CT scans of patients in a referral hospital for COVID-19 during the pandemic. The CT scans were obtained using a 16-row multidetector scanner (NeuViz 16 Classic CT, Neusoft Corporation, Shenyang, China; http://www.neusoftmedical.com/) with the following parameters: (1) $120 \mathrm{kVp}$, (2) $40 \mathrm{~mA}$, (3) $1.5 \mathrm{~mm}$ collimation, (4) 1.35:1 pitch, (5) a sharp kernel (B80f), (6) reconstruction matrix of $512 \times 512$, (7) slice thickness of $1.0 \mathrm{~mm}$, and (8) high-spatial-resolution algorithm. All images were analyzed by two experienced chest radiologists with more than ten years of experience, who were blinded to the clinical data.

The lung lesion features, including the number of involved lobes and the distribution of lesions in different lobes, were evaluated in each patient. We considered the lingula as a separate lobe from the left upper lobe. A scoring system was used to quantitatively evaluate the pulmonary involvement of all lesions, based on the involved location (17). The scoring criteria were typical chest CT findings for COVID-19 pneumonia (bilateral, multifocal rounded, and peripheral ground glass opacities and consolidations) (18). We analyzed the initial chest CT scans of the patients and scored the lung involvement based on two scoring methods of TLI and modified TLI.

For evaluating the TLI score, each of the six lung lobes was visually scored from 0 to 4 : 0 , no involvement; $1,1-25 \%$ involvement (minimal); 2, 26 - 50\% involvement (mild); 3, 51 - 75\% involvement (moderate); and 4, $\geq 76 \%$ involvement (severe). The TLI score was the sum of each individual lobe score, ranging from 0 (no involvement) to 24 (maximum involvement) (16). Since lobar volume was not considered, we defined the modified TLI score to determine if it can increase the accuracy of prognostic prediction; the lobar involvement score was multiplied by five for the lower lobes, by three for the upper lobes, and by two for the middle lobe and lingula. The modified TLI score was the sum of values, ranging from 0 (no involvement) to 80 (maximum involvement).

To evaluate the demographic characteristics, we classified the patients into four groups: (1) group A, patients admitted to the wards, but not intubated or expired; (2) group B, patients admitted to the ICU, but not intubated or expired; (3) group C, patients intubated, but not expired; and (4) group D, patients who expired. Considering some overlaps between the groups, we also applied another classification. Moreover, the patients were classified into groups to compare the sensitivity and specificity of TLI and the modified TLI scoring methods: Patients with ICU admission or without ICU admission; patients intubated during hospitalization or not intubated; and dead or alive patients.

\subsection{Statistical Analysis}

Statistical analyses were performed using IBM SPSS for Windows Version 20 (IBM Corp., Armonk, NY, USA). Quantitative data are presented as mean \pm standard deviation (minimum/maximum), and continuous data are presented as frequency and percentage. Chi-square $\left(\chi^{2}\right)$ test was used for categorical variables. The TLI score as a function of time was quantitatively measured using the 
SPSS curve estimation module. Besides, quantitative data were compared using independent sample $t$-test or MannWhitney U test, according to their normal distribution. Kruskal-Wallis test was used for comparing the TLI and modified TLI scores in different patient groups. Moreover, a receiver operating characteristic (ROC) curve analysis was performed to determine the clinical value of TLI and modified TLI CT scores and to measure the cut-off values for predicting the prognosis of COVID-19. P-value $<0.05$ was considered statistically significant in all analyses.

\section{Results}

\subsection{Characteristics and Chest CT Findings of COVID-19 Patients}

Of 207 patients, five were excluded due to pathological findings other than COVID-19 in their chest CT scans; also, one patient was excluded because of missing data. In total, 201 COVID-19 patients, aged between 13 and 97 years, were enrolled in this study. We categorized the patients into four independent groups: (1) group A, patients admitted to wards, but not intubated or expired (142 patients); (2) group B, patients admitted to ICU, but not intubated or expired (22 patients); (3) group C, patients intubated but not expired (13 patients); and (4) group D, patients who expired (24 patients) (Table 1 ).

The mean age of the patients was $54.8 \pm 16.9$ years in group A, $55.3 \pm 18.8$ years in group B, $38.3 \pm 18.6$ years in group $C$, and $67.8 \pm 10.7$ years in group $D$. The mean age of the patients was significantly different between the groups $(\mathrm{P}=0.001$ ) (Table 1). Among 201 patients, 125 (62.2\%) were male, and 76 (37.8\%) were female. In group A, $55.6 \%$ of the patients were male, and $44.4 \%$ were female. Among patients in group B, $68.2 \%$ were male, and $31.8 \%$ were female. In group C, all patients were male (100\%), and in group D, 75 and $25 \%$ were male and female, respectively. The patient's gender was significantly different between the groups $(\mathrm{P}=$ 0.006) (Table 1).

Moreover, we studied the lung involvement patterns in chest CT scans. Bilateral lung involvement was observed in $92 \%$ of the patients, and $84 \%$ of the patients showed a peripheral distribution of lesions. The patterns of lung involvement were mixed ground glass opacities and consolidations (75.6\%), pure ground glass opacities (17\%), and pure consolidations (7.4\%). Other radiological findings included interlobular septal thickening (39.3\%), crazy paving pattern (25.8\%), halo sign (27.3\%), and reverse halo sign (12.4\%).

Based on the Kruskal-Wallis test, the mean TLI score was significantly different between the groups $(\mathrm{P}<0.001)$ (Table 1 ). The mean TLI score was 8.99 in group A, 9.40 in group $\mathrm{B}, 12.53$ in group $C$, and 16.08 in group $D$. The mean modified
TLI scores of the groups are presented in Table 1. The degree of involvement of each lobe is presented in Table 2. Minimal involvement had the highest frequency among different lobes. The right lower lobe was the most frequently involved lobe and also the most severely involved one (Table 2).

Comparison of the number of involved lobes and the patients' prognoses showed that there was no significant difference among patients admitted to ICUs and patients admitted to the hospital wards $(\mathrm{P}=0.06)$ (Table 3). However, intubated patients had a significantly higher number of involved lobes compared to non-intubated patients ( $P$ $=0.002$ ) (Table 3 ). Patients who expired had the highest number of involved lobes $(\mathrm{P}=0.001)$ (Table 3$)$.

We also compared the TLI and modified TLI scores in the paired groups, including patients with ICU admission $(n=55)$ versus patients without ICU admission $(n=146)$; patients intubated during hospitalization $(n=37)$ versus patients not intubated $(\mathrm{n}=164)$; and patients who expired $(n=24)$ versus patients who survived $(n=177)$. The TLI and modified TLI scores were significantly higher in patients with ICU admission compared to patients without ICU admission $(\mathrm{P}=0.002)$. Intubated patients in comparison with non-intubated patients and expired patients in comparison with alive patients had significantly higher TLI and modified TLI scores $(\mathrm{P}<0.001)$ (Table 4$)$.

In the paired groups, there was no significant association between gender and mortality $(\mathrm{P}=0.168)$, whereas the difference in the male-to-female ratio was significant between the ICU admission $(\mathrm{P}=0.001)$ and intubation ( $\mathrm{P}$ $=0.003$ ) groups. Besides, the sensitivity and specificity of TLI and modified TLI scores for the prediction of ICU admission, intubation, and death were estimated using the ROC curve analysis and calculation of the area under the ROC curve (AUC) (Figures 1 - 3). The AUC showed no significant difference between the TLI and modified TLI scores in any of the groups (Figures 1-3).

\subsection{Diagnostic Ability of Chest CT Findings}

The sensitivity and specificity of TLI scores in patients with ICU admission, intubation, and death prognosis are presented in Table 5. The AUC was 0.65 (95\% CI: 0.55 - 0.74; $\mathrm{P}<0.001$ ) for patients admitted to the ICUs (Figure 1). The ROC curve analysis showed that the TLI score of 10.5 had $63 \%$ sensitivity and $63 \%$ specificity for ICU admission $(\mathrm{P}=0.001)$ (Table 5). Also, the AUC for intubation was 0.76 (95\% CI: 0.66 - $0.85 ; \mathrm{P}<0.001$ ) (Figure 2). The TLI score for the prediction of intubation was 11.5 , with $70.3 \%$ sensitivity and $70 \%$ specificity $(\mathrm{P}=0.001)$ (Table 5). The TLI scores for the prediction of death in COVID-19 patients are presented in Figure 3. The AUC attributed to death was 0.81 (95\% CI: 0.72 - 0.90; P < 0.001). The TLI score of 11.5 showed $79 \%$ sensitivity and $68 \%$ 


\begin{tabular}{|c|c|c|}
\hline Variables & Values & P-value \\
\hline $\operatorname{Age}(y)$ & & $0.001^{c}$ \\
\hline Total $(n=201)$ & $55.4 \pm 17.6$ & \\
\hline Group A $(\mathrm{n}=142)$ & $54.8 \pm 16.9$ & \\
\hline Group B $(n=22)$ & $55.3 \pm 18.8$ & \\
\hline Group C $(n=13)$ & $38.3 \pm 18.6$ & \\
\hline Group D $(n=24)$ & $67.8 \pm 10.7$ & \\
\hline Gender (male/female) & & $0.006^{\mathrm{d}}$ \\
\hline Total $(\mathrm{n}=201)$ & $125(62.2) / 76(37.8)$ & \\
\hline Group A $(\mathrm{n}=142)$ & $79(55.6) / 63(44.4)$ & \\
\hline Group B $(n=22)$ & $15(68.2) / 7(31.8)$ & \\
\hline Group C $(n=13)$ & $13(100) / 0(0)$ & \\
\hline Group D $(n=24)$ & $18(75) / 6(25)$ & \\
\hline Total lung involvement (TLI) score & & $<0.001^{\mathrm{e}}$ \\
\hline Group A $(n=142)$ & $8.99 \pm 4.83$ & \\
\hline Group B $(n=22)$ & $9.40 \pm 5.50$ & \\
\hline Group C $(n=13)$ & $12.53 \pm 7.38$ & \\
\hline Group D $(n=24)$ & $16.08 \pm 5.43$ & \\
\hline Modified total lung involvement (TLI) score & & $<0.001^{\mathrm{e}}$ \\
\hline Group A $(n=142)$ & $32.14 \pm 16.60$ & \\
\hline Group B $(n=22)$ & $33.13 \pm 18.23$ & \\
\hline Group C $(n=13)$ & $44.23 \pm 24.53$ & \\
\hline Group D $(n=24)$ & $55.58 \pm 18.32$ & \\
\hline
\end{tabular}

${ }^{\text {a }}$ Group A, patients who were admitted to hospital wards, but not intubated or expired; Group B, patients who were admitted to ICUs, but not intubated or expired; Group C, patients who were intubated, but did not expire; Group D, patients who expired.

${ }^{b}$ Values are expressed as mean \pm SD and No. (\%).

${ }^{\mathrm{c}} \mathrm{P}$-value is calculated by one-way ANOVA test.

d $P$-value is calculated by chi-square test.

${ }^{\mathrm{e}} \mathrm{P}$-value is calculated by Kruskal-Wallis test.

Table 2. The Frequency of Lobar Involvement in the Patients' Chest CT Scans ${ }^{\text {a }}$

\begin{tabular}{|c|c|c|c|c|c|}
\hline \multirow{2}{*}{ Lobar involvement } & \multicolumn{5}{|c|}{ Number of involved lobes (percentage) based on the severity of involvement ${ }^{b}$} \\
\hline & No involvement & Minimal involvement & Mild involvement & Moderate involvement & Severe involvement \\
\hline \multicolumn{6}{|l|}{ Upper lobes } \\
\hline Right upper lobe & $23(11.4)$ & $89(44.2)$ & $53(26.3)$ & $22(10.9)$ & $14(7)$ \\
\hline Left upper lobe & $30(14.9)$ & $94(46.7)$ & $43(21.4)$ & $22(10.9)$ & $12(5.9)$ \\
\hline \multicolumn{6}{|l|}{ Lower lobes } \\
\hline Right lower lobe & $11(5.4)$ & $60(29.8)$ & $46(22.8)$ & $46(22.8)$ & $38(18.9)$ \\
\hline Left lower lobe & $19(9.4)$ & $62(30.8)$ & $56(27.8)$ & $30(14.9)$ & $34(16.9)$ \\
\hline Lingula & $43(21.4)$ & $77(38.3)$ & $39(19.4)$ & $28(13.9)$ & $14(7)$ \\
\hline Middle lobe & $37(18.4)$ & $85(42.3)$ & $47(23.3)$ & $22(10.9)$ & $10(5)$ \\
\hline
\end{tabular}

${ }^{\text {a }}$ Values are expressed as No. (\%).

${ }^{\mathrm{b}}$ Each lobe was visually scored from 0 to 4: 0, no involvement; 1, 1-25\% involvement (minimal); 2, 26 - 50\% involvement (mild); 3, 51 - 75\% involvement (moderate); and

$4, \geq 76 \%$ involvement (severe) 
Table 3. The Number of Involved Lobes Among Patients in Each Group ${ }^{\text {a }}$

\begin{tabular}{ccc}
\hline Patient groups & Total number of involved lobes & Minimum -Maximum \\
\hline ICU admission & & P-value $^{\mathbf{b}}$ \\
No $(\mathrm{n}=146)$ & $5.08 \pm 1.52$ & 0.067 \\
Yes $(\mathrm{n}=55)$ & $5.47 \pm 1.23$ & $1-6$ Lobes \\
\hline Intubation & & 0.002 \\
\hline No $(\mathrm{n}=164)$ & $5.07 \pm 1.51$ & $0-6$ Lobes \\
Yes $(\mathrm{n}=37)$ & $5.70 \pm 1.05$ & $1-6$ Lobes \\
Death & & 0.001 \\
No $(\mathrm{n}=177)$ & $5.08 \pm 1.52$ & $0-6$ Lobes \\
Yes $(\mathrm{n}=24)$ & $5.95 \pm 0.20$ & $5-6$ Lobes \\
\hline
\end{tabular}

Abbreviation: ICU, intensive care unit.

a Values are expressed as mean \pm SD unless otherwise indicated.

${ }^{\mathrm{b}} \mathrm{P}$-value is calculated using Mann-Whitney $\mathrm{U}$ test.

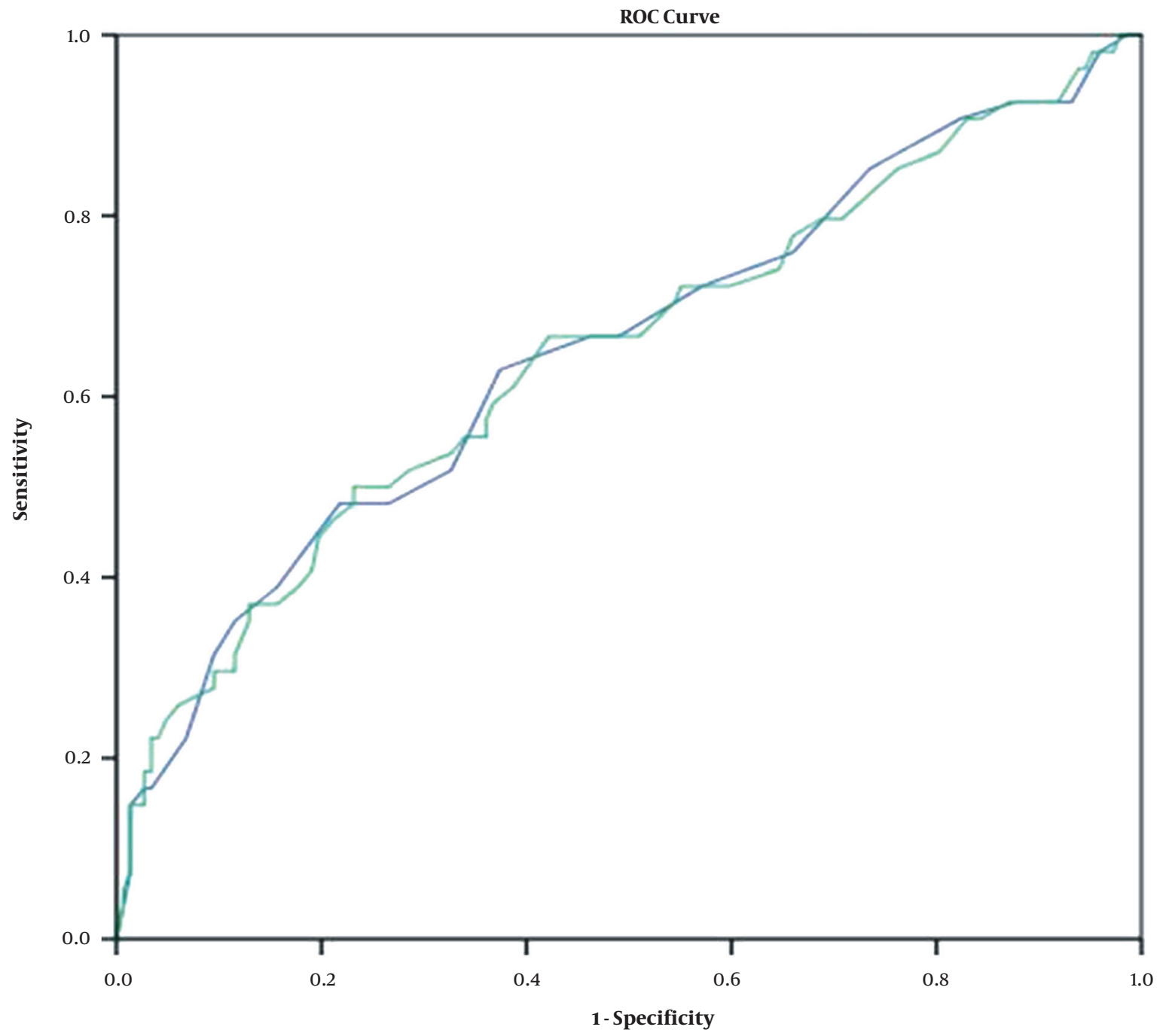

Figure 1. Total lung involvement (TLI) and modified TLI scores in ICU-admitted COVID-19 patients (blue line: TLI score; green line: modified TLI score). The ROC analysis showed that the area under curve (AUC) was 65\% (95\% CI: 55 - 74\%) for the TLI score and 64\% (95\% CI: 55 - 73\%) for the modified TLI score. 


\begin{tabular}{|c|c|c|c|}
\hline Patient groups & TLI score & Modified TLI score & P-value ${ }^{b, c}$ \\
\hline ICU admission & & & 0.002 \\
\hline No $(n=146)$ & $9.24 \pm 5.05$ & $32.96 \pm 17.30$ & \\
\hline Yes $(n=55)$ & $12.4 \pm 6.45$ & $43.45 \pm 21.67$ & \\
\hline Intubation & & & $<0.001$ \\
\hline No $(n=164)$ & $9.048 \pm 4.88$ & $32.28 \pm 16.77$ & \\
\hline Yes $(n=37)$ & $14.83 \pm 6.32$ & $51.59 \pm 21.10$ & \\
\hline Death & & & $<0.001$ \\
\hline No $(n=177)$ & $9.30 \pm 5.16$ & $33.15 \pm 17.65$ & \\
\hline Yes $(n=24)$ & $16.08 \pm 5.43$ & $55.58 \pm 18.32$ & \\
\hline
\end{tabular}

Abbreviation: ICU, intensive care unit; $\mathrm{N}$, number of related patients.

${ }^{\mathrm{a}}$ Values are expressed as mean $\pm \mathrm{SD}$.

${ }^{\mathrm{b}} \mathrm{P}$-value is calculated based on the difference in the TLI score column.

${ }^{\mathrm{C}} \mathrm{P}$-value is calculated using Mann-Whitney U test.

specificity, while the TLI score of 14.5 showed $62 \%$ sensitivity and $84 \%$ specificity. Also, the TLI score of 18.5 showed $96 \%$ specificity for the prediction of death in COVID-19 patients $(\mathrm{P}=0.001)$ (Table 5).

\section{Discussion}

COVID-19 pneumonia has various prognoses in different patients. Patients with severe or critical COVID-19 have a poor prognosis and a higher mortality rate compared to normal COVID-19 patients (19, 20). Among different methods used for studying the clinical manifestations of COVID-19, chest CT imaging can be helpful in understanding COVID-19 and improving its clinical diagnosis and management.

Several clinical and laboratory prognostic factors have been suggested as the predisposing factors for COVID-19 pneumonia, including age and underlying medical conditions. Critical cases and patients with severe symptoms are normally older ( $>55$ years), and patients with an underlying disease (chronic pulmonary, cardiovascular, or cerebrovascular disease and diabetes) commonly have a worse prognosis $(11,14,21)$. Sun et al. showed that depletion of lymphocytes and eosinophils and increased liver function tests, lactate dehydrogenase (LDH) level, albumin level, inflammatory markers (CRP), erythrocyte sedimentation rate (ESR), and ferritin are associated with COVID19 severity (9). It has been also proposed that the elevated serum levels of NT-proBNP, IL-6, and procalcitonin were associated with the severity of COVID-19 $(12,13)$.

A recent study showed that the elevated ratio of WAL/normal pattern of the lung in CT scan was associated with a better prognosis of COVID-19. For instance, patients with a WAL percentage $<73 \%$ were more prone to disease progression, ICU admission, or death (15). The present study evaluated the prognostic significance of quantitative chest CT imaging for COVID-19 patients. The TLI score and a new scale (modified TLI) were evaluated and compared between different groups of patients. We found that there was no significant difference between the TLI and modified TLI scoring methods for COVID-19. Both scales showed approximately similar sensitivity and specificity for predicting the prognosis of ICU admission, intubation, and death (Figures 1-3).

There was no significant association between gender and mortality, while the difference in the ratio of men to women was significant in the ICU admission and intubation groups. Unlike previous studies $(14,22)$, male sex could be considered as a risk factor for ICU admission or intubation in COVID-19 patients, based on the present results. However, this finding may be attributed to the higher frequency of COVID-19 in males than females in Iran (23). Moreover, evaluation of the number of involved lobes and prognosis showed that the highest number of involved lobes was associated with a higher mortality rate. A similar study on prognostic prediction via chest CT scan found that the odds ratio of death prognosis was higher in patients with higher CT scores and more involved lobes (24).

We assessed the TLI and modified TLI scores to quantify lung involvement and evaluate its correlation with the prognosis of COVID-19. There was a significant difference in the TLI and modified TLI scores between different prognostic groups $(\mathrm{P}<0.001)$. There was an overlap in the TLI and modified TLI scores in different groups. However, patients without ICU admission had significantly lower TLI and modified TLI scores compared to the other groups. We also measured the sensitivity and specificity of the scoring methods in the groups. At the TLI score of 11.5, with sensitivity and specificity of 70.3 and $70 \%$, respectively (AUC $=76 \%$; 95\% CI: 66 - 85\%), the patients were intubated.

Besides, the AUC for death was 81\% (95\% CI: 72 - 90\%), 


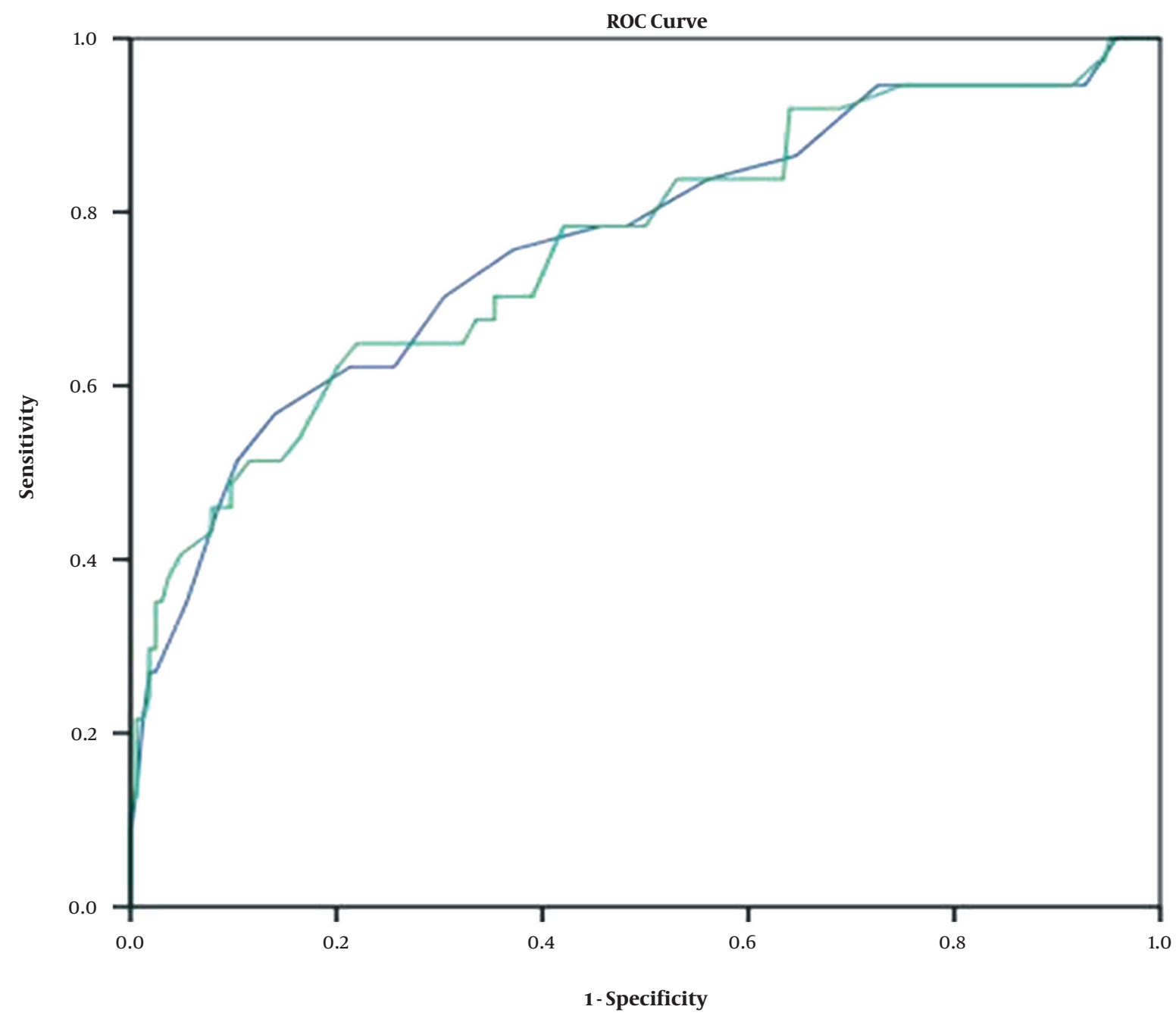

Figure 2. Total lung involvement (TLI) and modified TLI scores of intubated COVID-19 patients (blue line: TLI score; green line: modified TLI score). The ROC analysis showed that the area under curve (AUC) of TLI and modified TLI scores was 76\% (95\% CI: $66-85 \%)$.

Table 5. Evaluation of the Sensitivity and Specificity of the Total Lung Involvement (TLI) Score in Prognosis of COVID-19 Patients ${ }^{a}$

\begin{tabular}{|c|c|c|c|c|c|}
\hline \multirow{2}{*}{ Prognosis } & \multicolumn{5}{|c|}{ Measures } \\
\hline & AUC & 95\% CI & TLI score & Sensitivity (\%) & Specificity (\%) \\
\hline ICU admission & 0.650 & $0.55-0.74$ & 10.5 & 63 & 63 \\
\hline Intubation & 0.763 & $0.66-0.85$ & 11.5 & 70.3 & 70 \\
\hline \multirow{3}{*}{ Death } & \multirow{3}{*}{0.813} & \multirow{3}{*}{$0.72-0.90$} & 11.5 & 79 & 68 \\
\hline & & & 14.5 & 62 & 84 \\
\hline & & & 18.5 & 29 & 96 \\
\hline
\end{tabular}

Abbreviations: AUC, area under the curve; CI, confidence interval (lower bound-upper bound); ICU, intensive care unit; TLI, total lung involvement.

${ }^{a}$ The test result variable (s) have at least one tie between the positive actual state group and the negative actual state group. Statistics may be biased.

and the TLI score of 11.5 showed $79 \%$ sensitivity and $68 \%$ specificity. The sensitivity and specificity were measured to be 62 and $84 \%$ for the TLI score of 14.5 and 29 and $96 \%$ for the TLI score of 18.5 , respectively. Patients with intubation and patients who expired had higher TLI scores compared to patients who were only admitted to a ward or ICU with- 


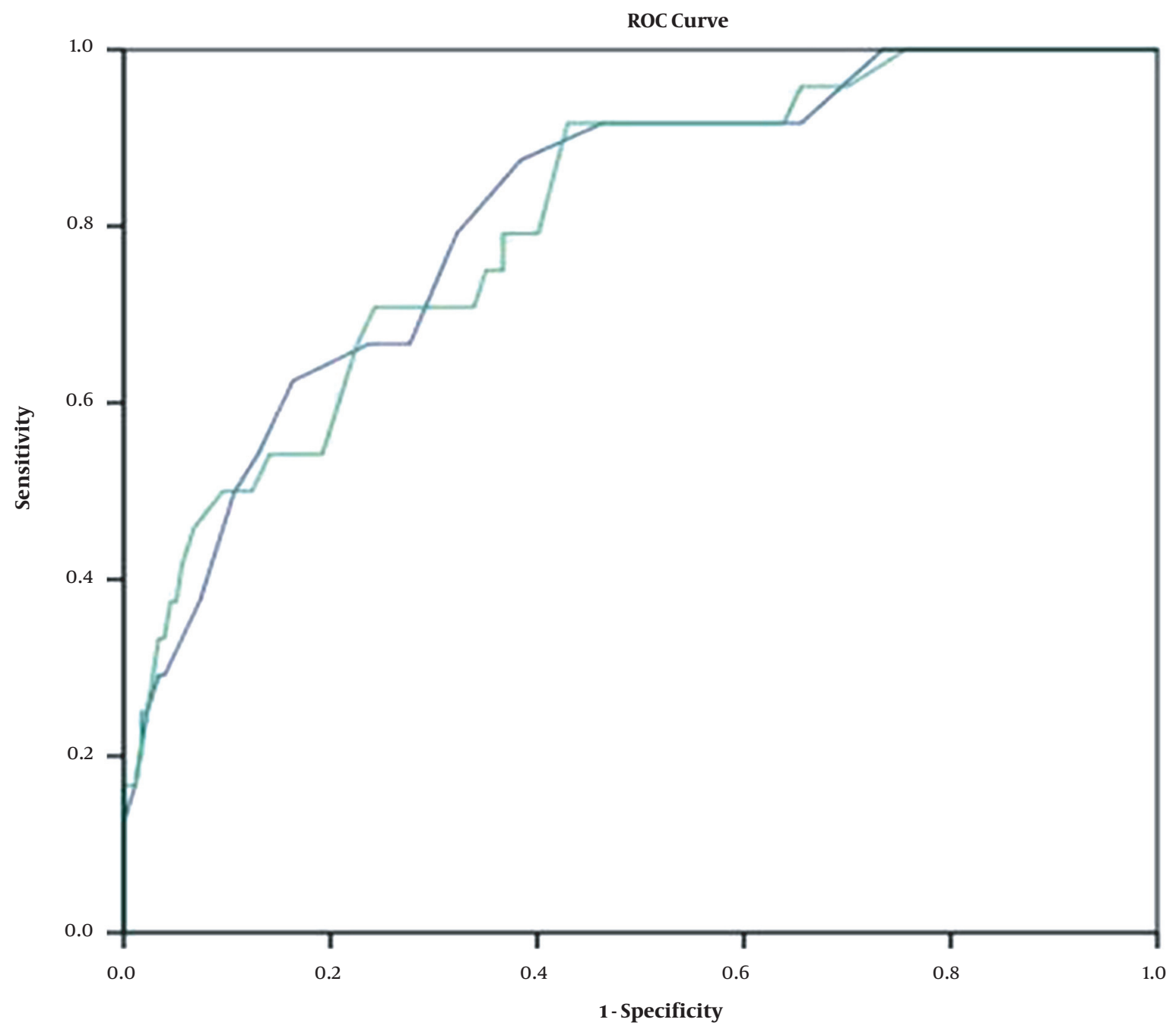

Figure 3. Total lung involvement (TLI) and modified TLI scores for mortality in COVID-19 patients (blue line: TLI score; green line: modified TLI score). The ROC analysis showed that the area under curve (AUC) was 81\% (95\% CI: 72 - 90\%) for the TLI score and 80\% (95\% CI: 71 - 89\%) for the modified TLI score.

out intubation. Our results are similar to the findings of a study by Li et al. (25), which found that the AUC of the total lung involvement score for diagnosing severe/critical COVID-19 was 0.918 (95\% CI: 0.843 - 0.994$)$.

Since COVID-19 has different clinical outcomes, and there are limited medical facilities for in-hospital management, application of a method for early prediction of prognosis can be helpful during the pandemic. Despite evaluating a large number of COVID-19 patients in the present study, this study had several limitations. First, we did not apply both qualitative and quantitative chest CT indicators to identify the CT characteristics of all cases. Second, we only analyzed the initial chest CT scans of patients; there- fore, more follow-up imaging is suggested in future research. Third, we did not assess the correlation of clinical features and outcomes with chest CT scan features. Therefore, evaluation of both qualitative and quantitative chest CT findings, follow-up chest CT scan, and assessment of clinical symptoms and medical history are recommended in future cohort studies.

In conclusion, the severity of COVID-19 is associated with chest CT indicators, including TLI and the number of involved lobes. Patients with higher TLI scores in chest CT scan had a greater likelihood of severe disease, leading to intubation or death. The TLI scoring system seems to have a prognostic value in determining the need for ICU admis- 
sion and vital healthcare planning in critical situations, such as the current COVID-19 pneumonia pandemic.

\section{Acknowledgments}

This study was supported by the Vice-Chancellor for Research of Mashhad University of Medical Sciences, Mashhad, Iran (thesis research protocol number: 990033). The authors would like to thank the Clinical Research Development Unit of Ghaem Hospital for their contribution to data analysis.

\section{Footnotes}

Authors' Contribution: Study concept and design, B.A.; Acquisition of data, AR.T., A.T., M.M., and M.F.; Analysis and interpretation of data, P.L. and B.A.; Drafting of the manuscript, M.M. and AR.T.; Critical revision of the manuscript for important intellectual content, M.M.; Statistical analysis: M.E.; Administrative, technical, and material support, M.F.; and Study supervision, B.A.

Conflict of Interests: All authors declare that there is no conflict of interest.

\section{Ethical Approval: IR.MUMS.REC.1399.029.}

Funding/Support: This study was supported by Mashhad University of Medical Sciences.

Informed Consent: There was no need to obtain informed consent forms from the participants because of the retrospective nature of this study.

\section{References}

1. Huang C, Wang Y, Li X, Ren L, Zhao J, Hu Y, et al. Clinical features of patients infected with 2019 novel coronavirus in Wuhan, China. Lancet. 2020;395(10223):497-506. doi: 10.1016/S0140-6736(20)30183-5. [PubMed: 31986264]. [PubMed Central: PMC7159299].

2. World health Organization. Advice on the use of masks in the community, during home care and in health care settings in the context of the novel coronavirus (2019-nCoV) outbreak: Interim guidance. Geneva, Switzerland: World health Organization; 2020, [updated 29th Jan 2020]. Available from: https://apps.who.int/iris/bitstream/handle/10665/330987/WHOnCov-IPC_Masks-2020.1-eng.pdf?sequence $=1 \&$ isAllowed $=y$.

3. World health Organization. Advice on the use of masks in the community, during home care, and in health care settings in the context of COVID-19: Interim guidance. Geneva, Switzerland: World health Organization; 2020, [updated 19th Mar 2020]. Available from: https: //apps.who.int/iris/bitstream/handle/10665/331493/WHO-2019nCoV-IPC_Masks-2020.2-eng.pdf?sequence=14\&isAllowed=y.

4. Chan JF, Yuan S, Kok KH, To KK, Chu H, Yang J, et al. A familial cluster of pneumonia associated with the 2019 novel coronavirus indicating person-to-person transmission: A study of a family cluster. Lancet. 2020;395(10223):514-23. doi:10.1016/S0140-6736(20)30154-9. [PubMed: 31986261]. [PubMed Central: PMC7159286].
5. Ai T, Yang Z, Hou H, Zhan C, Chen C, Lv W, et al. Correlation of chest CT and RT-PCR testing for Coronavirus disease 2019 (COVID19) in China: A report of 1014 cases. Radiology. 2020;296(2):E32-40. doi: 10.1148/radiol.2020200642. [PubMed: 32101510]. [PubMed Central: PMC7233399].

6. Fang Y, Zhang H, Xie J, Lin M, Ying L, Pang P, et al. Sensitivity of chest CT for COVID-19: Comparison to RT-PCR. Radiology. 2020;296(2):E1157. doi: 10.1148/radiol.2020200432. [PubMed: 32073353]. [PubMed Central: PMC7233365].

7. Kanne JP, Little BP, Chung JH, Elicker BM, Ketai LH. Essentials for radiologists on COVID-19: An update-radiology scientific expert panel. Radiology. 2020;296(2):E113-4. doi: 10.1148/radiol.2020200527. [PubMed: 32105562]. [PubMed Central: PMC7233379].

8. Yang Y, Yang M, Yuan J, Wang F, Wang Z, Li J, et al. Laboratory diagnosis and monitoring the viral shedding of SARS-CoV-2 infection. Innovation (NY). 2020;1(3):100061. doi: 10.1016/j.xinn.2020.100061. [PubMed: 33169119]. [PubMed Central: PMC7609236].

9. Sun Y, Dong Y, Wang L, Xie H, Li B, Chang C, et al. Characteristics and prognostic factors of disease severity in patients with COVID-19: The Beijing experience. J Autoimmun. 2020;112:102473. doi: 10.1016/j.jaut.2020.102473. [PubMed: 32439209]. [PubMed Central: PMC7180376].

10. Wang L, He W, Yu X, Hu D, Bao M, Liu H, et al. Coronavirus disease 2019 in elderly patients: Characteristics and prognostic factors based on 4-week follow-up. J Infect. 2020;80(6):639-45. doi: 10.1016/j.jinf.2020.03.019. [PubMed: 32240670]. [PubMed Central: PMC7118526].

11. Guo W, Li M, Dong Y,Zhou H, Zhang Z, Tian C, et al. Diabetes is a risk factor for the progression and prognosis of COVID-19. Diabetes Metab Res Rev.2020. e3319. doi:10.1002/dmrr.3319. [PubMed: 32233013]. [PubMed Central: PMC7228407].

12. Gao L, Jiang D, Wen XS, Cheng XC, Sun M, He B, et al. Prognostic value of NT-proBNP in patients with severe COVID-19. Respir Res. 2020;21(1):83. doi: 10.1186/s12931-020-01352-w. [PubMed: 32293449]. [PubMed Central: PMC7156898].

13. Liu F, Li L, Xu M, Wu J, Luo D, Zhu Y, et al. Prognostic value of interleukin-6, C-reactive protein, and procalcitonin in patients with COVID-19. J Clin Virol. 2020;127:104370. doi: 10.1016/j.jcv.2020.104370. [PubMed: 32344321]. [PubMed Central: PMC7194648].

14. Li K, Wu J, Wu F, Guo D, Chen L, Fang Z, et al. The clinical and chest CT features associated with severe and critical COVID-19 pneumonia. Invest Radiol. 2020;55(6):327-31. doi: 10.1097/RLI.0000000000000672. [PubMed: 32118615]. [PubMed Central: PMC7147273].

15. Colombi D, Bodini FC, Petrini M, Maffi G, Morelli N, Milanese G, et al. Well-aerated lung on admitting chest CT to predict adverse outcome in COVID-19 pneumonia. Radiology. 2020;296(2):E86-96. doi: 10.1148/radiol.2020201433. [PubMed:32301647]. [PubMed Central: PMC7233411].

16. Hani C, Trieu NH, Saab I, Dangeard S, Bennani S, Chassagnon G, et al. COVID-19 pneumonia: A review of typical CT findings and differential diagnosis. Diagn Interv Imaging. 2020;101(5):263-8. doi: 10.1016/j.diii.2020.03.014. [PubMed: 32291197]. [PubMed Central: PMC7129663].

17. Chang YC, Yu CJ, Chang SC, Galvin JR, Liu HM, Hsiao $\mathrm{CH}$, et al. Pulmonary sequelae in convalescent patients after severe acute respiratory syndrome: evaluation with thin-section CT. Radiology. 2005;236(3):1067-75. doi: 10.1148/radiol.2363040958. [PubMed: 16055695].

18. Simpson S, Kay FU, Abbara S, Bhalla S, Chung JH, Chung M, et al. Radiological society of north America expert consensus document on reporting chest CT findings related to COVID-19: Endorsed by the society of thoracic radiology, the American College of Radiology, and RSNA. Radiol Cardiothorac Imaging. 2020;2(2). e200152. doi: 10.1148/ryct.2020200152. [PubMed: 33778571]. [PubMed Central: PMC7233447]. 
19. Bernheim A, Mei X, Huang M, Yang Y, Fayad ZA, Zhang N, et al. Chest CT findings in Coronavirus disease-19 (COVID-19): Relationship to duration of infection. Radiology. 2020;295(3):200463. doi: 10.1148/radiol.2020200463. [PubMed: 32077789]. [PubMed Central: PMC7233369].

20. Wang C, Horby PW, Hayden FG, Gao GF. A novel coronavirus outbreak of global health concern. Lancet. 2020;395(10223):470-3. doi: 10.1016/S0140-6736(20)30185-9. [PubMed: 31986257]. [PubMed Central: PMC7135038].

21. Yang X, Yu Y, Xu J, Shu H, Xia J, Liu H, et al. Clinical course and outcomes of critically ill patients with SARS-CoV-2 pneumonia in Wuhan, China: A single-centered, retrospective, observational study. Lancet Respir Med. 2020;8(5):475-81. doi: 10.1016/S2213-2600(20)30079-5. [PubMed: 32105632]. [PubMed Central: PMC7102538].

22. Chen N, Zhou M, Dong X, Qu J, Gong F, Han Y, et al. Epidemiological and clinical characteristics of 99 cases of 2019 novel coronavirus pneumonia in Wuhan, China: A descriptive study. Lancet.
2020;395(10223):507-13. doi: 10.1016/S0140-6736(20)30211-7. [PubMed: 32007143]. [PubMed Central: PMC7135076].

23. Nikpouraghdam M, Jalali Farahani A, Alishiri G, Heydari S, Ebrahimnia M, Samadinia H, et al. Epidemiological characteristics of coronavirus disease 2019 (COVID-19) patients in Iran: A single center study. J Clin Virol. 2020;127:104378. doi: 10.1016/j.jcv.2020.104378. [PubMed: 32353762]. [PubMed Central: PMC7172806].

24. Sabri A, Davarpanah AH, Mahdavi A, Abrishami A, Khazaei M, Heydari S, et al. Novel coronavirus disease 2019: Predicting prognosis with a computed tomography-based disease severity score and clinical laboratory data. Pol Arch Intern Med. 2020;130(7-8):629-34. doi: 10.20452/pamw.15422. [PubMed: 32500700].

25. Li K, Fang Y, Li W, Pan C, Qin P, Zhong Y, et al. CT image visual quantitative evaluation and clinical classification of coronavirus disease (COVID-19). Eur Radiol. 2020;30(8):4407-16. doi: 10.1007/s00330-02006817-6. [PubMed: 32215691]. [PubMed Central: PMC7095246]. 